\title{
Immunotherapy for Recurrent Pregnancy Loss: A Review
}

\author{
Paprikar Manoj ${ }^{1}$, Paprikar Monika ${ }^{2}$ \\ ${ }^{1}$ Classified Specialist (Obs \& Gyn), Military Hospital, Devlali, Nashik, Maharashtra-422401 \\ ${ }^{2}$ Associate Professor, Dept of Dravyaguna, Ashvin Rural Ayurved College and Hospital, Manchi Hills, \\ Sangamner, Maharashtra-413714 \\ Corresponding Author: Paprikar Manoj
}

\begin{abstract}
Modern medicine has made extraordinary progress in the last few decades. But little success has been achieved in the management of recurrent pregnancy losses and implantation failures post in vitro fertilization. It results in extreme duress and financial burden on the couples seeking treatment. To prevent such pregnancy losses a lot of new therapies are offered to them. Immunotherapies like Allogenic Lymphocyte Immunization, Corticosteroid therapy, Intravenous Immunoglobulin, etc., are being researched and tried.

We searched current literature for evidence pertaining to all the different immunotherapies for treatment. We studied trials, meta-analyses and review articles on it. This review has summarized the current status of research and evidence on all these therapies.

We concluded that the causation and mechanism of alteration of immune system has been well documented in most of the therapies. Clinical evidence indicating definite benefit is conflicting and at times lacking. More research and studies need to be done to validate these therapies for routine clinical use.
\end{abstract}

Keywords: Recurrent pregnancy loss, Recurrent implantation failure, Immunotherapy, Abortions

\section{INTRODUCTION}

Modern era in medicine has brought about a significant improvement in the fertility potential of the couple. Pathbreaking discoveries and technologies have made things possible that were only in the realm of science fiction couple of decades ago. In spite of these advances there remains an area wherein not much has changed. It is recurrent pregnancy losses and implantation failures post In Vitro Fertilization Embryo-Transfer (IVF-ET). These failures and losses are extremely traumatic to the patients as well as to the clinicians treating them. Various therapies have been tried to mitigate this problem and lot is being experimented.

Miscarriage is defined as the spontaneous loss of pregnancy before the foetus reaches viability. The term therefore includes all pregnancy losses from the time of conception until 24 weeks of gestation. ${ }^{[1]}$ In 1990, Sirrat stated that "commonly accepted definition of recurrent miscarriage is three or more consecutive miscarriages." [2] From then on, various societies have debated on the most acceptable definition. There has been a gradual shift from three or more consecutive losses to only two nonconsecutive losses. American Society of Reproductive Medicine describes two nonconsecutive documented miscarriages whereas Royal College of Obstetrics and Gynaecology states three consecutive losses before the period of viability. ${ }^{[1,3]}$

\section{Causes of recurrent pregnancy loss}

The overall per cycle conception rate was $40 \%$ over the first 12 months. The rate of clinical pregnancy was $30 \%$. Approximately one third of all the conceptions detected by urinary hCG assay 
failed to survive to delivery. More than two thirds of these losses occurred before the pregnancy had been clinically recognized.

Several risk factors have been linked with Recurrent Miscarriages (RM); the most studied are genetic disorders, congenital or acquired alterations in the uterine anatomy, hormonal diseases, obesity and antiphospholipid syndrome. Other causes of RM are immunological disorders (auto and alloimmune), hereditary thrombophilia, infection, environmental factors and causes related to male factors, or a combination of these factors. ${ }^{[4]}$ Almost $50 \%$ of cases remain unexplained and the treatment for alloimmune causes unclear. So immunotherapy has been proposed in the treatment of unexplained recurrent pregnancy loss (RPL).

\section{Immunology of pregnancy}

Disturbed immune balance at the maternal-fetal interface has been postulated as one of the most important causes of RPL and IVF outcomes. Elevated Natural Killer (NK) activity in peripheral blood or in the intrauterine environment, dysregulated cytokines, antiphospholipid antibodies and other antibodies, imbalanced $\mathrm{T}$ helper (Th) 1 and Th2 cell reaction, elevated Th1/Th2 cell ratio and the sharing of human leukocyte antigen alleles between the couple are important factors. ${ }^{[5]}$

For this maternal-fetal interface to adapt optimally certain changes take place. The maternal cytotoxic adaptive immune response is diminished while the regulatory adaptive immunity is enhanced. The innate immunity remains intact to protect against infections and interact with fetal tissue and to promote successful implantation, placentation and pregnancy.

HLA plays a crucial role in this. An abnormally formed blastocyst exposes the paternally derived antigens and invites rejection via humoral factors and uterine NK cells. Altered expressions of HLA in the form of HLA-E, HLA-F and HLA-G have a protective effect against the immune response from $\mathrm{T}$ cells, macrophages and uterine NK cells. B7H1 protein expressed on syncytiotrophoblast, indoleamine 2,3 dioxygenase (IDO) which depletes tryptophan, have an effect on suppressing and inactivating $\mathrm{T}$ cells. Placenta produces progesterone, prostaglandins E2 and cytokines such as interleukin-10 and interleukin-4, all of which are antiinflammatory. Soluble thymic stromal lymphopoietin (TSLP) is secreted by trophoblasts and stimulates decidual dendritic cells to produce IL-10 and chemokine (C-C motif) ligand 17 (CCL17) which induces $\mathrm{T}$-helper cell differentiation. Lower levels of TSLP have been found in patients suffering from RPL. ${ }^{[6]}$

\section{Immunotherapy in RPL}

\section{Allogenic Lymphocyte Immunization}

International literature has been debating the efficacy and success of Allogenic Lymphocyte Immunization. The first double-blind, randomized study was published by Mowbray et al. in 1985. ${ }^{\text {[7] }}$ Since then, there have been many ups and downs and controversies about this therapy. After numerous RCTs, the first metanalysis was published in 1992, by Fraser et al., who did not detect an improvement in the rate of live births in treated patients. ${ }^{[8]}$

Subsequently, in 1994, The Recurrent Miscarriage Immunotherapy Trialist Group published the results of their meta-analysis, which was a multi-center trial. The group concluded that immunotherapy with lymphocytes improved the rate of live births in patients with the history of RM (three or more consecutive miscarriages), with no more than one live birth with any partner [odds ratio (OR) 1.16, confidence interval (CI) 1.04-1.34], or no more than one live birth with the current partner (OR 1.21, CI 1.04-1.37). They observed worsening rates in patients with autoimmune disorders. ${ }^{[9]}$

In 2014, Cochrane database in their update to the 2001 meta-analysis concluded that the treatment effect on the live birth rate was not significant, with OR of 1.22 and CI 
of $0.89-1.69$. They further said that there was no improvement in the rate of live births observed when assessing the use of immunotherapy with unrelated donor lymphocytes, with OR of 1.39 and CI of $0.68-2.82$. $^{[10]}$ This meta-analysis was criticized for inclusion of results by Ober et al., which had negated the positive claims of all others. The Ober trial was criticized on many aspects like storage of blood for several hours before preparation, lack of post-immunization control of immunomodulation, different routes of administration of immunotherapy, etc. The results were subsequently withdrawn by them.

Liu et al. published a new metaanalysis in the American Journal of Reproductive Immunology aiming to correct all the flaws and criticisms of Cochrane meta-analysis. They demonstrated that immunization with lymphocytes promoted a significant improvement in the rate of live births: $77.8 \%$ in the group of treated women, when compared with the rate of $46.1 \%$ in the control group, with OR of 4.02 and CI of 3.23-5.00. On analysis of the subgroups of patients based on which protocol of immunization with lymphocytes was used, they concluded that when immunization with lymphocytes was performed before and during pregnancy, the results were better. And observed that the results were better when the concentration of lymphocytes used was lower than $100 \mathrm{x}$ $10^{6}$ per application (OR 5.25, CI 4.16-6.64), when compared with a concentration greater than $100 \times 10^{6}$ per application (OR 1.52, CI $1.04-2.22)^{[11]}$

\section{Corticosteroid Therapy}

Glucocorticoids are known to have anti-inflammatory properties and therefore have been used for treating autoimmune disorders like systemic lupus erythematosus and antiphospholipid antibody syndrome. They have also been tried in treating pregnancy losses due to it. Presence of glucocorticoid receptors on NK cells has made immunotherapy look more promising.
A study by Quenby et al. demonstrated that prednisolone effectively reduces NK cells at the level of endometrium from 14 to $9 \% .^{[12]}$

Tang et al. reported a higher birth rate $(12 / 20)$ in prednisolone treated patients vis-a-vis placebo $(8 / 20)$, but did not reach statistical significance. ${ }^{[13]}$ Gomaa et al. found a higher on-going pregnancy rate $(52 / 74)$ in those treated with prednisolone with low-dose aspirin and unfractionated heparin as against those with only low-dose aspirin and unfractionated heparin. ${ }^{[14]}$

A meta-analysis in 2015 by Dan et al. concluded that prednisolone therapy improves pregnancy outcomes in women with idiopathic RM (live birth rate: RR $1.58,95 \%$ CI 1.23-2.02; successful pregnancy outcome: RR 7.63, 95\% CI 3.7115.69; miscarriage rate: RR $0.42,95 \%$ CI 0.28-0.61). Their meta-analysis revealed a non-significant effect of prednisolone on pregnancy outcome during intracytoplasmic sperm injection (ICSI) cycles (pregnancy rate: RR 1.02, 95\% CI 0.84-1.24; clinical pregnancy rate: RR $1.01,95 \%$ CI $0.82-1.24$; implantation rate: RR $1.04,95 \%$ CI $0.85-$ 1.28). ${ }^{[15]}$ This again shows conclusively the positive effect of prednisolone therapy in patients with recurrent miscarriages but maybe not so in ICSI cycles.

Cooper et al. in 2019, carried out a retrospective cohort study of the effect of prednisolone on endometrial uterine NK cell concentrations and pregnancy outcome in women with reproductive failure. This study showed a relatively high prevalence of raised uNK cells in women with recurrent reproductive failure and confirmed the effect of prednisolone on reducing the uNK cell concentrations. They found however no evidence for a significant beneficial effect for prednisolone therapy on pregnancy outcomes. This is definitely a ray of hope in the right direction but needs more studies to confirm the findings. ${ }^{[16]}$

A triple therapy consisting of aspirin, prednisone and multivitamin showed that the rate of successful pregnancy was similar between the triple therapy $(89.6 \%)$ and control (92.3\%) groups 
$(\mathrm{P}=0.343)$. The rate of successful treatment was higher in the triple therapy $(86.3 \%)$ than in the control $(53.3 \%)$ group $(\mathrm{P}<0.001)$. In multivariate logistic regression analysis, triple therapy was associated with higher odds of successful treatment, whereas the number of spontaneous pregnancy losses was associated with lower odds of successful treatment. They thus concluded that the triple therapy of aspirin, prednisone, and multivitamin was a good treatment option for women with unexplained recurrent spontaneous abortions. ${ }^{[17]}$

So, currently there are studies which show that corticosteroids do have an effect on reducing the NK cell concentration that is raised in recurrent miscarriage patients. But treatment offers a doubtful increase in the live birth rate although it reduces the concentration of the NK cells. More RCTs and variations in the dose and duration of treatment may yield favorable results in the near future.

\section{Intravenous Immunoglobulin (IVIG)}

Intravenous immunoglobulin is prepared by extracting $\mathrm{IgG}$ fractions from normal blood plasma of blood donors as well as trace amounts of $\operatorname{IgA}$ and $\operatorname{IgM}$. In the late 1980s, passive immunization with intravenous immunoglobulin (IVIG) was used to treat RSA, and was reported to have beneficial effects. The underlying mechanism might be associated with neutralization of autoantibodies in the circulatory system, inhibition of natural killer cell, attenuation of complementmediated cytotoxicity and release of regulatory T lymphocytes. ${ }^{[18]}$

Coulam et al. in 1995 reported a significant higher birth rate in treated patients $^{[19]}$, but later an Italian multicentre trial reported no difference among primary recurrent miscarriages treated with IVIG $(16 / 22)(68 \%)$ vs, placebo $(19 / 24){ }^{[20]}$

Later, there were multiple studies which reported a significant benefit of treatment in secondary recurrent pregnancy losses but not in primary. These trials were conducted by Stephenson et al., Christiansen et al. and systematic review by Hutton et al. ${ }^{[21-23]}$

Meta-analysis conducted by Wang et al. of 11 studies showed that the difference in the live birth rate between the IVIG treatment and placebo groups was on the margin of significance $(\mathrm{RR}=1.25,95 \% \mathrm{CI}$ 1.00 to $1.56, \mathrm{P}=0.05$ ). Both cumulative and trial sequential meta-analyses indicated potential beneficial effect of IVIG but the evidence was inconclusive. Subgroup analysis showed that the live birth rate in primary $(\mathrm{RR}=0.88,95 \% \mathrm{CI} 0.71$ to 1.07 ) and secondary $(\mathrm{RR}=1.26$, 95\% CI 0.99 to 1.61) RSA patients was not significantly different between the IVIG and placebo groups. Live birth rate was significantly different when IVIG was administered before conception ( $\mathrm{RR}=1.67,95 \%$ CI 1.30 to $2.14, \mathrm{P}<0.0001$ ) but not after implantation $(\mathrm{RR}=1.10,95 \%$ CI 0.93 to 1.29$){ }^{[24]}$

Thus, presently there is evidence which points towards some beneficial effect with IVIG, more clearly for secondary RPL. But it needs more trials and evidence for confirmation.

\section{Intralipid}

Intravenous intralipid (IVI), a fatbased emulsion of soybean oil, glycerine, phospholipids, egg, and poly unsaturated fatty acids, has been evaluated as a potential intervention to suppress the maternal immunological response, by reducing monocyte endothelial adhesion and proinflammatory cytokine generation. Although the exact mechanism remains unclear, several studies support its role in downregulating the uNK cell macrophages pro-inflammatory mediators especially $\mathrm{T} 1$ helper cells. ${ }^{[25,26]}$ This seems to be mediated by the interaction between the intralipid fatty acids of and the uNK cell peroxisome proliferator-activated receptors which decrease uNK cytotoxicity through reduced secretion of interferon- $\gamma$, demonstrated both in vivo and in vitro. ${ }^{[27,28]}$

Rimmer et al. conducted a systematic review and meta-analysis to 
evaluate the effectiveness of IVI in improving pregnancy rates in women with history of recurrent implantation failure (RIF). All trials used 20\% IVI solution at the time of embryo transfer compared to normal saline infusion or no intervention (routine care). The IVI group had a higher chance of clinical pregnancy (172 vs 119, risk ratio $[\mathrm{RR}] 1.55,95 \%$ confidence interval [CI] 1.16-2.07, $\mathrm{I}^{2} 44.2 \%$ ) and live birth (132 vs 73 , RR 1.83 , 95\% CI $1.42-$ $\left.2.35, \mathrm{I}^{2} 0 \%\right)$ post treatment compared to no intervention. They concluded that there is limited evidence to support the use of IVI at the time of embryo transfer in women with the history of RIF. ${ }^{[29]}$

Canella et al. carried out a review of all the studies from 2008 to 2020 that used Lipid emulsion therapy (LET) for treating RPL and RIF. They observed that sixty per cent of the reviewed studies obtained satisfactory results demonstrating that LET contributed to decreasing NK cell activity in patients with RPL and RIF. Twenty per cent mentioned that they achieved satisfactory and significant results with the LET, although the data for NK cell activity were not addressed in the results and discussion, that would have been interesting to confirm whether there had been a significant reduction of those cells. Twenty per cent did not obtain satisfactory results with LET. ${ }^{[30]}$

These studies emphasize that although there is a lot of promise, substantial and conclusive evidence is yet not available to prescribe the therapy with complete confidence.

\section{Tumor Necrosis Factor Alpha (TNF- $\alpha)$ Inhibitors}

TNF controls cyclooxygenases that affect blastocyst implantation, vascular permeability of the endometrium and uterine deciduation. ${ }^{[31]}$ High levels of TNF$\alpha$ have been implicated in such pregnancy complications as infection and fetal growth retardation and have been linked to early and unexplained spontaneous abortions. ${ }^{[32,33]}$ So, potentially drugs that block TNF- $\alpha$, would have a positive beneficial effect in treatment.

El-Far et al. reported a fall in the percentage of [CD3+ CD56+ NK T cells] and TNF- $\alpha$ in peripheral blood in patients with recurrent miscarriage after treatment with sildenafil citrate. They demonstrated that intravaginal sildenafil citrate tablets can reduce vasoconstriction by increasing blood flow through relaxation of uterine arteries as indicated from measured PI in these patients. They concluded that intravaginal sildenafil citrate tablets used as suppositories might be a novel, interesting, safe anti-abortive option in the treatment of threatened miscarriage in patients with a history of recurrent miscarriage. ${ }^{[34]}$

Seventy-five pregnancies in patients with a history of RSA were retrospectively evaluated by Winger et al. The population was divided into three groups: group I: 21 patients treated with AC (anticoagulants), group II: 37 patients treated with $\mathrm{AC}$ and IVIG, and group III: 17 patients treated with AC, IVIG and the TNF inhibitor Etanercept or Adalimumab. The live birth rate was 19\% $(4 / 21)$ in group I, 54\% (20/37) in group II and $71 \%(12 / 17)$ in group III. There was significant improvement in pregnancy outcome in group II versus group I $(\mathrm{P}=0.0127)$ and in group III versus group I $(\mathrm{P}=0.0026)$. The live birth rate in group III compared to group II was not significantly different $(\mathrm{P}=0.3723)$. In women with RSA, addition of IVIG or a TNF- $\alpha$ inhibitor with IVIG to the AC regimen appears to improve live birth rates compared to treatment with anticoagulation or IVIG with anticoagulation alone.

Sildenafil enhances activity of nitric oxide which induces proinflammatory cytokines including TNF- $\alpha$. It is a potent activator of NK cells. Ohams et al. investigated the influence of sildenafil and etanercept on the level of TNF- $\alpha$ in women with recurrent miscarriages. Patients treated with sildenafil had a higher level of TNF- $\alpha$ after therapy, whereas those with etanercept showed a statistically significant decrease in the levels. ${ }^{[35]}$ This clearly shows that the 
levels of TNF- $\alpha$ can be modulated with drugs which can have a beneficial effect on pregnancy continuation. More trials in this direction would prove to be beneficial.

\section{Granulocyte-Colony Stimulating Factor (G-CSF)}

Granulocyte colony-stimulating factor (G-CSF) is a hematopoietic-specific cytokine produced by bone marrow cells, stromal cells, fibroblasts, and macrophages, although several nonhematopoietic cell types, such as endothelial cells, placental cells, trophoblasts and granulosa-lutein cells, also express G-CSF receptors. G-CSF plays a role in embryo implantation and the continuation of pregnancy by temporarily suppressing immune response through its effects on lymphocytes, macrophages and $\mathrm{T}$ helper-2 cells. ${ }^{[3]}$

A randomised controlled trial with G-CSF or placebo was conducted by Scarpellini and Sbracia in sixty-eight women with unexplained primary RM, all with at least four consecutive miscarriages. Patients were randomized for s.c. treatment with G-CSF $(\mathrm{n}=35) \quad(1 \mathrm{microg} / \mathrm{kg} / \mathrm{day})$ starting on the sixth day after ovulation, or with placebo $(\mathrm{n}=33)$. Results in the group treated with G-CSF, 29 out of $35(82.8 \%)$ women delivered a healthy baby, whereas in the placebo group, this figure was only 16 out of $33(48.5 \%)(\mathrm{P}=0.0061, \mathrm{OR}=5.1$; $95 \%$ CI 1.5-18.4). Significantly higher betahCG levels were found in gestation weeks 5-9 in women treated with G-CSF versus placebo $(\mathrm{P}<0.001){ }^{[37]}$

Santjohanser et al. performed a retrospective cohort study in patients undergoing assisted reproductive treatment (ART) with known RM analysing the possible benefits of G-CSF. 127 patients (199 cycles) with RM (at least 2 early miscarriages) 49 (72 cycles) receiving G-CSF and 78 (127 cycles) controls receiving either no medication (subgroup 1) or Cortisone, intravenous immunoglobulins or low molecular weight heparin (subgroup 2). The LBR of patients and the subgroups differed significantly
(G-CSF $32 \%$, subgroup $113 \%$, subgroup 2 $14 \%)$. ${ }^{[38]}$

Another study by Boxer et al., identified 224 pregnancy events in women diagnosed with chronic neutropenia and identified a decrease in abortion rates with no adverse side effects. ${ }^{[39]}$

A randomized, double-blind, placebo controlled clinical trial involving 150 women with a history of unexplained recurrent pregnancy loss was conducted at 21 sites by Eapen et al., co-ordinated by University of Birmingham. Recombinant human G-CSF administered in the first trimester of pregnancy did not improve outcomes among women with a history of unexplained recurrent pregnancy loss. ${ }^{[40]}$

After looking at all these studies and their conflicting data, benefit of G-CSF remains doubtful and it should not be routinely used outside research trials.

\section{DISCUSSION}

Recurrent pregnancy losses and implantation failures post IVF are very bothersome and traumatic both to the patient couple as well as to the treating physician. Moreover, the treatment involved is very expensive. Some of the therapies being offered are experimental in nature. The data that is available is conflicting and hence does not give a clear-cut direction to the treating physician. Sometimes, as the couple is under extreme duress, these therapies are prescribed without proper counselling and justification, adding to the cost of the treatment. This review has summarized all the evidence that is currently available.

$$
\text { Allogenic Lymphocyte }
$$

Immunization offers hope for improving live birth rates and data is reassuring. More studies to arrive at exact dose and frequency need to be carried out to strengthen the evidence. Corticosteroids definitely influence the NK cells which play a crucial role in pregnancy failure. But data is lacking that shows a conclusive benefit on pregnancy outcome. Combination therapy along with aspirin and multivitamins may pave the way for definitive treatment in the 
future, and studies need to validate this. Some benefit of IVIG treatment has been definitely documented. Hence it can be used for improving outcomes.

Intralipid therapy convincingly modulates the uNK but studies have demonstrated only a marginal effect on live birth rate with conflicting results. TNF- $\alpha$ inhibitors have a well- documented effect but it has yet not shown to have a definite clinical improvement. G-CSF has limited trials that have shown some benefits but some studies have negated the results. So, it requires further research.

\section{CONCLUSION}

The failure of pregnancies in recurrent miscarriages and implantation failures take a heavy toll on the patients and the physician in terms of both- efforts and finances. Although many immunotherapies are being tried with varying success, and appear promising, yet data is conflicting and still not concrete. An approach that uses combination of multiple therapies might be the way forward in managing this vexing medical problem.

\section{Acknowledgement: None}

\section{Conflict of Interest: None}

\section{Source of Funding: None}

\section{REFERENCES}

1. The Investigation and Treatment of Couples with Recurrent First-trimester and Second-trimester Miscarriage Greentop Guideline No. 17. 2011;

2. Stirrat GM. Recurrent miscarriage I: definition and epidemiology. The Lancet [Internet]. 1990 Sep 15 [cited 2021 Sep 29];336(8716):673-5. Available from: http://www.thelancet.com/article/0140673 69092159F/fulltext

3. Practice Committee of the American Society for Reproductive Medicine $\mathrm{T}$. Evaluation and treatment of recurrent pregnancy loss: a committee opinion. Fertility and Sterility [Internet]. 2012 Nov 1 [cited 2021 Sep 18];98(5):1103-11.
Available

from:

http://www.fertstert.org/article/S00150282 12007017/fulltext

4. Garrido-Gimenez C, Alijotas-Reig J. Recurrent miscarriage: causes, evaluation and management. Postgraduate Medical Journal [Internet]. 2015 Mar 1 [cited 2021 Sep 28];91(1073):151-62. Available from:

https://pmj.bmj.com/content/91/1073/151

5. Achilli C, Duran-Retamal M, Saab W, et al. The role of immunotherapy in in vitro fertilization and recurrent pregnancy loss: a systematic review and meta-analysis. Fertility and Sterility [Internet]. 2018 Nov 1 [cited 2021 Sep 29];110(6):1089-100. Available from: http://www.fertstert.org/article/S00150282 18305697/fulltext

6. Farquharson RG, Stephenson MD. Early pregnancy. Second ed. New Delhi: Cambridge University Press; 2017. 247248.

7. Mowbray JF, Liddell H, Underwood Jennifer L, et al. Controlled trial of treatment of recurrent spontaneous abortion by immunisation with paternal cells. The Lancet [Internet]. 1985 Apr 27 [cited 2021 Sep 28];325(8435):941-3. Available from: http://www.thelancet.com/article/S014067 3685917234/fulltext

8. Fraser EJ, Grimes DA, Schulz KF. Immunization as therapy for recurrent spontaneous abortion: A review and metaanalysis. Vol. 82, Obstetrics and Gynecology. 1993. p. 854-9.

9. Group TRMIT, Coulam CB, Clark DA, Collins J, et al. Worldwide Collaborative Observational Study and Meta-Analysis on Allogenic Leukocyte Immunotherapy for Recurrent Spontaneous Abortion1. American Journal of Reproductive Immunology [Internet]. 1994 Sep 1;32(2):55-72. Available from: https://doi.org/10.1111/j.16000897.1994.tb01095.x

10. Wong LF, Porter TF, Scott JR. Immunotherapy for recurrent miscarriage. Cochrane Database of Systematic Reviews [Internet]. 2014 Oct 21 [cited 2021 Sep 28];2014(10). Available from: 
https://www.cochranelibrary.com/cdsr/doi /10.1002/14651858.CD000112.pub3/full

11. Liu Z, Xu H, Kang X, et al. Allogenic Lymphocyte Immunotherapy for Unexplained Recurrent Spontaneous Abortion: A Meta-Analysis. American Journal of Reproductive Immunology. 2016 Dec 1;76(6):443-53.

12. Quenby S, Kalumbi C, Bates M, et al. Prednisolone reduces preconceptual endometrial natural killer cells in women with recurrent miscarriage. Fertility and Sterility [Internet]. 2005 Oct 1 [cited 2021 Sep 22];84(4):980-4. Available from: http://www.fertstert.org/article/S00150282 05014275/fulltext

13. Tang A-W, Alfirevic Z, Turner MA, et al. A feasibility trial of screening women with idiopathic recurrent miscarriage for high uterine natural killer cell density and randomizing to prednisolone or placebo when pregnant. Advanced Access publication on April [Internet]. 2013 [cited 2021 Sep 22];28(7):1743-52. Available from: https://academic.oup.com/humrep/article/ 28/7/1743/613604

14. Gomaa MF, Elkholy AG, et al. Combined oral prednisolone and heparin versus heparin: the effect on peripheral NK cells and clinical outcome in patients with unexplained recurrent miscarriage. A double-blind placebo randomized controlled trial. Archives of Gynecology and Obstetrics 2014 290:4 [Internet]. 2014 May 13 [cited 2021 Sep 22];290(4):75762. Available from: https://link.springer.com/article/10.1007/s 00404-014-3262-0

15. Dan S, Wei W, Yichao S, et al. Effect of Prednisolone Administration on Patients with Unexplained Recurrent Miscarriage and in Routine Intracytoplasmic Sperm Injection: A Meta-Analysis. American Journal of Reproductive Immunology. 2015 Jul 1;74(1):89-97.

16. Cooper S, Laird SM, Mariee N, et al. The effect of prednisolone on endometrial uterine NK cell concentrations and pregnancy outcome in women with reproductive failure. A retrospective cohort study. Journal of Reproductive Immunology. 2019 Feb 1;131:1-6.

17. $\mathrm{Ou} \mathrm{H}, \mathrm{Yu}$ Q. Efficacy of aspirin, prednisone, and multivitamin triple therapy in treating unexplained recurrent spontaneous abortion: A cohort study. International Journal of Gynecology \& Obstetrics [Internet]. 2020 Jan 1 [cited 2021 Sep 21];148(1):21-6. Available from:

https://onlinelibrary.wiley.com/doi/full/10 .1002/ijgo.12972

18. Schwab I, Nimmerjahn F. Intravenous immunoglobulin therapy: how does IgG modulate the immune system? Nature Reviews Immunology 2013 13:3 [Internet]. $2013 \mathrm{Feb} 15$ [cited 2021 Sep 29];13(3):176-89. Available from: https://www.nature.com/articles/nri3401

19. Coulam Cb, Krysa L, Stern Jj, Bustillo M. Intravenous Immunoglobulin for Treatment of Recurrent Pregnancy Loss. American Journal of Reproductive Immunology [Internet]. 1995 Dec 1 [cited 2021 Sep 23];34(6):333-7. Available from:

https://onlinelibrary.wiley.com/doi/full/10 .1111/j.1600-0897.1995.tb00960.x

20. Perino A, Vassiliadis A, Vucetich A, et al. Short-term therapy for recurrent abortion using intravenous immunoglobulins: Results of a double-blind placebocontrolled Italian study. Human Reproduction. 1997;12(11):2388-92.

21. Stephenson MD, Dreher K, Houlihan E, $\mathrm{Wu}$ V. Prevention of Unexplained Recurrent Spontaneous Abortion Using Intravenous Immunoglobulin: A Prospective, Randomized, DoubleBlinded, Placebo-Controlled Trial. American Journal of Reproductive Immunology [Internet]. 1998 Feb 1 [cited 2021 Sep 23];39(2):82-8. Available from: https://onlinelibrary.wiley.com/doi/full/10 .1111/j.1600-0897.1998.tb00339.x

22. Christiansen OB, Pedersen B, Rosgaard A, Husth M. A randomized, double-blind, placebo-controlled trial of intravenous immunoglobulin in the prevention of recurrent miscarriage: evidence for a therapeutic effect in women with secondary recurrent miscarriage. Human 
Reproduction [Internet]. 2002 Mar 1 [cited 2021 Sep 23];17(3):809-16. Available from: https://academic.oup.com/humrep/article/ 17/3/809/642363

23. Hutton B, Sharma R, Fergusson D, et al. Use of intravenous immunoglobulin for treatment of recurrent miscarriage: a systematic review. BJOG: An International Journal of Obstetrics \& Gynaecology [Internet]. 2007 Feb 1 [cited 2021 Sep 23];114(2):134-42. Available from:

https://onlinelibrary.wiley.com/doi/full/10 .1111/j.1471-0528.2006.01201.x

24. Wang SW, Zhong SY, Lou LJ, et al. The effect of intravenous immunoglobulin passive immunotherapy on unexplained recurrent spontaneous abortion: a metaanalysis. Reproductive BioMedicine Online. 2016 Dec 1;33(6):720-36.

25. Granato D, Blum S, Rössle C, et al. Effects of Parenteral Lipid Emulsions With Different Fatty Acid Composition on Immune Cell Functions In Vitro. Journal of Parenteral and Enteral Nutrition [Internet]. 2000 Mar 1 [cited 2021 Sep 27];24(2):113-8. Available from: https://onlinelibrary.wiley.com/doi/full/10 $.1177 / 0148607100024002113$

26. Mayer K, Meyer S, Reinholz-Muhly M, et al. Short-Time Infusion of Fish Oil-Based Lipid Emulsions, Approved for Parenteral Nutrition, Reduces Monocyte Proinflammatory Cytokine Generation and Adhesive Interaction with Endothelium in Humans. The Journal of Immunology [Internet]. 2003 Nov 1 [cited 2021 Sep 27];171(9):4837-43. Available from:

https://www.jimmunol.org/content/171/9/ 4837

27. Roussev RG, Ng SC, Coulam CB. Natural Killer Cell Functional Activity Suppression By Intravenous Immunoglobulin, Intralipid and Soluble Human Leukocyte Antigen-G. American Journal of Reproductive Immunology [Internet]. 2007 Apr 1 [cited 2021 Sep 27];57(4):262-9. Available from: https://onlinelibrary.wiley.com/doi/full/10 $.1111 / \mathrm{j} .1600-0897.2007 .00473 . \mathrm{x}$
28. Roussev RG, Acacio B, Ng SC, Coulam CB. ORIGINAL ARTICLE: Duration of Intralipid's Suppressive Effect on NK Cell's Functional Activity. American Journal of Reproductive Immunology [Internet]. 2008 Sep 1 [cited 2021 Sep 27];60(3):258-63. Available from: https://onlinelibrary.wiley.com/doi/full/10 $.1111 / \mathrm{j} .1600-0897.2008 .00621 . x$

29. Rimmer MP, Black N, Keay $S$, et al. Intralipid infusion at time of embryo transfer in women with history of recurrent implantation failure: A systematic review and meta-analysis. Journal of Obstetrics and Gynaecology Research. 2021 Jun 1;47(6):2149-56.

30. Canella PRBC, Barini R, Carvalho P de $\mathrm{O}$, Razolli DS. Lipid emulsion therapy in women with recurrent pregnancy loss and repeated implantation failure: The role of abnormal natural killer cell activity. Journal of Cellular and Molecular Medicine. 2021 Mar 1;25(5):2290-6.

31. Imseis HM, Zimmerman PD, Samuels P, Kniss DA. Tumour necrosis factor- $\alpha$ induces cyclo-oxygenase-2 gene expression in first trimester trophoblasts: Suppression by glucocorticoids and NSAIDs. Placenta. 1997;18(7):521-6.

32. Daher S, Fonseca F, Ribeiro OG, et al. Tumor necrosis factor during pregnancy and at the onset of labor and spontaneous abortion. European Journal of Obstetrics and Gynecology and Reproductive Biology. 1999;83(1).

33. XW Yu, CF Yan, H Jin, X Li. Tumor necrosis factor receptor 1 expression and early spontaneous abortion. International journal of gynaecology and obstetrics: the official organ of the International Federation of Gynaecology and Obstetrics [Internet]. 2005 [cited 2021 Sep 24];88(1):44-8. Available from: https://pubmed.ncbi.nlm.nih.gov/1561770 4/

34. El-Far M, El-Motwally AEG, Hashem IA, Bakry N. Biochemical role of intravaginal sildenafil citrate as a novel antiabortive agent in unexplained recurrent spontaneous miscarriage: First clinical study of four case reports from Egypt. 
Clinical Chemistry and Laboratory Medicine. 2009;47(11):1433-8.

35. Ohams M, Jerzak M, Górski A. Effects of sildenafil citrate and etanercept treatment on TNF- $\alpha$ levels in peripheral blood of women with recurrent miscarriage. Ginekologia Polska. 2015;86(7):520-4.

36. Moldenhauer LM, Keenihan SN, Hayball JD, Robertson SA. GM-CSF Is an Essential Regulator of $\mathrm{T}$ Cell Activation Competence in Uterine Dendritic Cells during Early Pregnancy in Mice. The Journal of Immunology. 2010 Dec 1;185(11):7085-96.

37. Scarpellini F, Sbracia M. Use of granulocyte colony-stimulating factor for the treatment of unexplained recurrent miscarriage: A randomised controlled trial. Human Reproduction. 2009;24(11): 2703-8.

38. Santjohanser C, Knieper C, Franz C, et al. Granulocyte-colony stimulating factor as treatment option in patients with recurrent miscarriage. Archivum Immunologiae et Therapiae Experimentalis. 2013 Apr;61(2):159-64.

39. Boxer LA, Bolyard AA, Marrero TM, et al. Outcomes of Pregnancies for Women with Severe Chronic Neutropenia with or without G-CSF Treatment. Blood. 2010 Nov 19;116(21):1490.

40. Eapen A, Joing M, Kwon P, et al. Recombinant human granulocyte- colony stimulating factor in women with unexplained recurrent pregnancy losses: A randomized clinical trial. Human Reproduction. 2019 Mar 1;34(3):424-32.

How to cite this article: Paprikar M, Paprikar M. Immunotherapy for recurrent pregnancy loss: a review. International Journal of Research and Review. 2021; 8(10): 83-92. DOI: https://doi.org/10.52403/ijrr.20211013 